\title{
Inhibition of late-stage autophagy synergistically enhances pyrrolo-1,5-benzoxazepine-6-induced apoptotic cell death in human colon cancer cells
}

\author{
LISA M. GREENE ${ }^{1}$, DEREK P. NOLAN ${ }^{1}$, DANIEL REGAN-KOMITO ${ }^{1}$, GIUSEPPE CAMPIANI ${ }^{2}$, \\ D. CLIVE WILLIAMS ${ }^{1}$ and DANIELA M. ZISTERER ${ }^{1}$ \\ ${ }^{1}$ School of Biochemistry and Immunology, Trinity Biomedical Sciences Institute, Trinity College Dublin, \\ Dublin 2, Ireland; ${ }^{2}$ European Research Centre for Drug Discovery and Development, I-53100 Siena, Italy
}

Received March 6, 2013; Accepted April 23, 2013

DOI: $10.3892 /$ ijo.2013.1989

\begin{abstract}
The pyrrolo-1,5-benzoxazepines (PBOXs) are a novel group of selective apoptotic agents displaying promising therapeutic potential in both ex vivo chemotherapy-refractory patient samples and in vivo murine carcinoma models. In this report, we present novel data concerning the induction of autophagy by the PBOXs in adenocarcinoma-derived colon cancer cells. Autophagy is a lysosome-dependent degradative pathway recently associated with chemotherapy. However, whether autophagy facilitates cell survival in response to chemotherapy or contributes to chemotherapy-induced cell death is highly controversial. Autophagy was identified by enhanced expression of LC3B-II, an autophagosome marker, an increase in the formation of acridine orange-stained cells, indicative of increased vesicle formation and electron microscopic confirmation of autophagic structures. The vacuolar $\mathrm{H}^{+}$ATPase inhibitor bafilomycin-A1 (BAF-A1) inhibited vesicle formation and enhanced the apoptotic potential of PBOX-6. These findings suggest a cytoprotective role of autophagy in these cells following prolonged exposure to PBOX-6. Furthermore, BAF-A1 and PBOX-6 interactions were determined to be synergistic and caspase-dependent. Potentiation of PBOX-6-induced apoptosis by BAF-A1 was associated with a decrease in the levels of the anti-apoptotic protein, Mcl-1. The data provide evidence that autophagy functions as a survival mechanism in colon cancer cells to PBOX-6-induced apoptosis and a rationale for the use of autophagy inhibitors to further enhance PBOX-6-induced apoptosis in colon cancer.
\end{abstract}

Correspondence to: Dr Daniela Zisterer, School of Biochemistry and Immunology, Trinity College, 152-160 Pearse Street, Dublin 2, Ireland

E-mail: dzistrer@tcd.ie

Key words: pyrrolo-1,5-benzoxazepines autophagy apoptosis

\section{Introduction}

The pyrrolo-1,5-benzoxazepines (PBOXs) were originally designed and synthesised as a novel series of peripheral-type benzodiazepine receptor (PBR) ligands (1). Subsequent studies demonstrated novel proapoptotic properties for the PBOXs that were independent of the PBR (2). Tubulin was later identified as the molecular target of a subset of the PBOX series (3). The tubulin targeting PBOXs demonstrated selective proapoptotic activity across a wide spectrum of human tumour cell types and tumour models whilst displaying minimal toxicity towards normal cells (4-8). The most potent member of the series PBOX-15 effectively induced apoptosis in ex vivo B cell chronic lymphocytic leukaemia and chronic myeloid leukaemia cells derived from patients displaying resistance to current first line therapies $(8,9)$. We recently described the anti-angiogenic activity of the PBOXs a property that may augment its antineoplastic properties in vivo (10). However, despite the promising anticancer and anti-angiogenic properties of the PBOXs some cell types display some inherent resistance to the compounds.

Previous studies in colon cancer models demonstrated activation of macroautophagy (hereafter referred to as autophagy) as a cytoprotective response to nutritional deprivation (11), hydrogen sulphide exposure (12) and chemotherapeutics commonly used in treatment of colon cancer (5-fluorouracil, topotecan, irinotecan) (13-15). Autophagy is defined as a catabolic mechanism mediating the recycling and turnover of cytoplasmic components in response to starvation or stress (16). Autophagy is frequently activated in tumour cells treated with chemotherapy, endocrine therapy and irradiation (17-21). Despite the copious advances in autophagy research the role played by autophagy in cell death and cell survival still remains highly controversial. It is postulated that autophagy constitutes an attempt to adapt to lethal stress imposed by chemotherapeutics as opposed to a cell death mechanism (18). However, some still argue for the existence of genuine autophagic cell death (22). Targeting autophagy by genetic or pharmacological means was shown to trigger the apoptotic pathway in several preclinal studies $(23,24)$. Furthermore, inhibition of autophagy sensitises cancer cells to chemotherapy (14), radiation (25) and immunotherapy (26). To 
date there are 19 ongoing clinical trials investigating the efficacy and toxicity of autophagy inhibitors as an adjuvant therapy for the treatment of solid and haematological cancers (27). The dual phosphatidylinositol 3-kinase/mammalian target of rapamycin inhibitor NVP-BEZ235 is currently undergoing phase I/II clinical trials for the treatment of advanced solid tumours (28) and in radiosensitisation (29). Also, the anti-malarial/autophagy inhibitor chloroquine is undergoing phase I/II clinical trials as a single agent and also in combination with various chemotherapeutics and/or radiation for a diverse range of solid tumours and leukaemia (30).

In this report, we investigate the possibility of cytoprotective macroautophagy (hereafter referred to as autophagy) as a means of continued cell survival despite prolonged exposure to the PBOXs in colon cancer cells. Manipulation of autophagy was also explored as a means of augmenting the pro-apoptotic properties of the PBOXs.

\section{Materials and methods}

Compounds. The pyrrolo-1,5-benzoxazepine compounds, 7-[(N,N-dimethylcarbamoyl)oxy]-6-(naphth-1-yl)pyrrolo[2,1-d] $[1,5]$ benzoxazepine (PBOX-6) and 4-acetoxy-5-(1-(naphthyl) naphtho[2,3-b]pyrrolo[2,1-d][1,4]oxazepine (PBOX-15) (Fig. 1A) were synthesised as described previously (1) and were prepared as $5 \mathrm{mM}$ stocks in ethanol and stored at $-20^{\circ} \mathrm{C}$. Bafilomycin-A1 (BAF-A1) was purchased from Sigma-Aldrich (Poole, UK). All general reagents unless stated otherwise were purchased from Sigma-Aldrich. The general caspase inhibitor Z-VAD-FMK was purchased from Merck Biosciences (Nottingham, UK).

Cell culture. CT-26 and Caco-2 were originally obtained from European Collection of Cell Cultures (Salisbury, UK). Cells were grown in DMEM Glutamax media supplemented with $50 \mathrm{U} / \mathrm{ml}$ penicillin and $50 \mu \mathrm{g} / \mathrm{ml}$ streptomycin. CT-26 media also contained $10 \%$ FBS, $1 \%$ non-essential amino acids (NEAA) and $100 \mathrm{mg} / \mathrm{l}$ sodium pyruvate. Caco-2 media contained $20 \%$ FBS and $1 \%$ NEAA. Cells were maintained at $37^{\circ} \mathrm{C}$ in $5 \% \mathrm{CO}_{2}$ in a humidified incubator. Cell culture materials were supplied from Gibco, Invitrogen Corp (Grand Island, NY, USA).

Cell proliferation assay. For cell viability assays cells, were seeded at $5 \times 10^{3}(\mathrm{CT}-26)$ or $1 \times 10^{4}$ (Caco-2) per well in 96-well flat-bottomed plates and allowed to attach for $24 \mathrm{~h}$. Subsequently, cells were then exposed to either medium alone, ethanol vehicle $[1 \%$ ethanol $(\mathrm{v} / \mathrm{v})]$ or serial dilutions of PBOX compounds (0.1-50 $\mu \mathrm{M})$. After $72 \mathrm{~h}$ the number of viable cells was estimated using the Alamar Blue assay (Invitrogen Corp, Carlsbad, CA, USA) according to the manufacturer's instructions. The results were expressed as the percentage cell viability relative to vehicle-treated control cells (100\%). Dose response curves were plotted and $\mathrm{IC}_{50}$ values were obtained using the commercial software package Prism (GraphPad Software Inc., La Jolla, CA, USA). Experiments were performed in triplicate on at least three separate occasions.

Cell cycle analysis. Cells in the log phase of growth were treated with relevant vehicle, drug or drug combination for the time indicated. After treatment, cells were fixed with $70 \%(\mathrm{v} / \mathrm{v})$ ethanol and stored at $-20^{\circ} \mathrm{C}$. Cells were then centrifuged and stained with PBS containing $0.5 \mathrm{mg} / \mathrm{ml}$ RNase and $0.15 \mathrm{mg} / \mathrm{ml}$ propidium iodide for $30 \mathrm{~min}$ at $37^{\circ} \mathrm{C}$. The PI fluorescence was measured on a linear scale using a FACSCalibur flow cytometer (Becton-Dickinson, San Jose, CA, USA). The amount of PI fluorescence is directly proportional to the amount of DNA present in each cell (6). Data collection was gated to exclude cell debris and cell aggregates. At least 10,000 cells were analysed per sample. All data were recorded and analysed using the CellQuest software (Becton-Dickinson).

Quantification of autophagy by flow cytometric analysis of acidic vesicular organelles (AVOs). The formation of acidic compartments was quantified by flow cytometric analysis of acridine orange stained cells. The intensity of the red fluorescence is proportional to the amount of acidity. Following treatment, cells were stained with acridine orange $(1 \mu \mathrm{g} / \mathrm{ml})$ for $15 \mathrm{~min}$ at $37^{\circ} \mathrm{C}$. BAF-A1 (Sigma-Aldrich) was dissolved in DMSO and added to the cells 45 min prior to the addition of acridine orange. Cells were then trypsinised and collected in phenol-red free medium. Green $(510-530 \mathrm{~nm})$ and red (>650 nm) fluorescence emission from $10^{4}$ cells illuminated with blue $(488 \mathrm{~nm})$ excitation light was measured with a CyAn ADP Flow Cytometry Analyzer (Beckman Coulter, Nyon, Switzerland). The red:green fluorescence ratio for individual cells was calculated using FlowJo software (Tree Star Inc., San Carlos, CA, USA).

Transmission electron microscopy (TEM). To morphologically examine PBOX-induced autophagy, CT-26 cells were exposed to ethanol vehicle $[0.2 \%(\mathrm{v} / \mathrm{v})]$ or PBOX-15 $(1 \mu \mathrm{M})$ for $48 \mathrm{~h}$. Adherent cells were harvested by trypsinisation, fixed for $1 \mathrm{~h}$ at room temperature in $4 \%$ paraformaldehyde, $2.5 \%$ glutaraldehyde, 0.125 M HEPES pH 7.5. After washing in PBS the cells were post-fixed in $2 \%$ osmium tetroxide solution and dehydrated in a series of aqueous ethanol solutions. Samples were embedded in epoxy resin. Ultrathin sections were cut on an ultramicrotome (RMC MTXL) and collected on 300 mesh copper grids. Each grid was stained with uranyl acetate and lead citrate and stored for ultrastructural examination. Ultrastructural examination was carried out in a Jeol 2100 transmission electron microscope operating at $100 \mathrm{kV}$. A number of images were obtained as a representative of each sample.

Western blot analysis. Cells were harvested in whole cell lysis buffer containing $62.5 \mathrm{mM}$ Tris (pH 6.8), $2 \%$ (w/v) SDS, $10 \%$ (v/v) glycerol, $0.00125 \%(\mathrm{w} / \mathrm{v})$ bromophenol blue and $50 \mathrm{mM}$ DTT. The proteins were separated on a polyacrylamide gel and transfered to PVDF membrane, probed overnight with the indicated primary antibody at $4^{\circ} \mathrm{C}$ and relevant HRP-conjugated secondary antibody for $1 \mathrm{~h}$ at room temperature. Rabbit anti-LC3B, antibeclin-1, anti-Mcl-1, lysosome-associated membrane protein 1 (LAMP-1) and anti- $\alpha$-actin were purchased from Cell Signaling (Danvers, MA, USA). The LC3B (microtubule-associated protein light chain 3) antibody used has a higher affinity for LC3B-II. Anti-PARP, anti-caspase-3, anti-Bcl-2, anti-Bcl-xL, anti-GAPDH and anti- $\alpha$-actin mAbs were purchased from Merck Biosciences. Immunoreactive bands were detected by autoradiography with enhanced chemiluminescence (Amersham Biosciences, Buckinghamshire, UK). 
A<smiles></smiles>

PBOX-6<smiles>CC(=O)OC1=C(c2cccc3ccccc23)Oc2cc3ccccc3cc2-n2cccc21</smiles>

PBOX-15

\section{B}
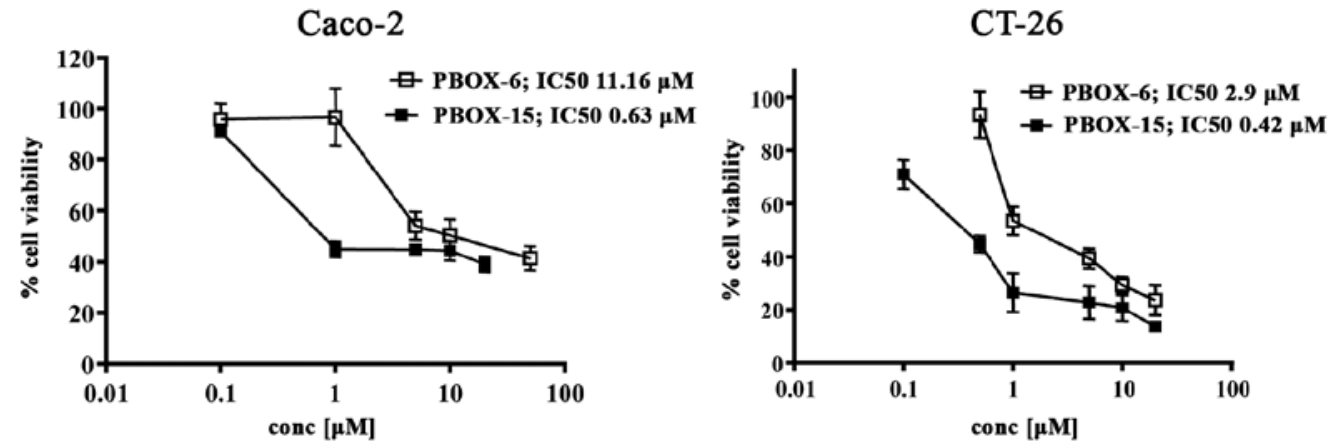

C
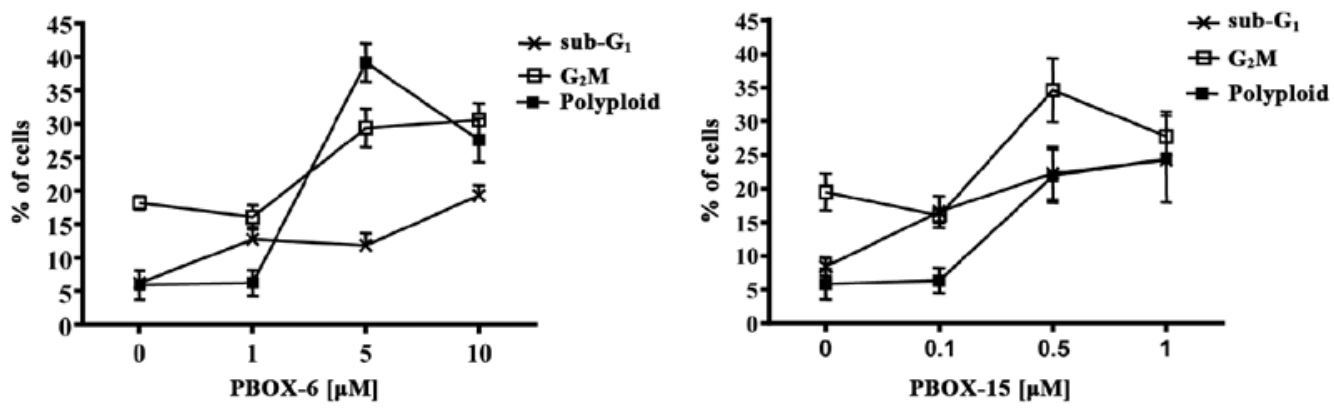

Figure 1. (A) Chemical structures of PBOX-6 and PBOX-15. (B) Alamar blue cell viability assays for cells exposed to the indicated concentration of PBOXs for $72 \mathrm{~h}$. The half-maximal inhibitory concentration $\left(\mathrm{IC}_{50}\right)$ for each compound in each cell line is indicated. (C) Flow cytometric analysis of propidium iodide stained cells after 48-h treatment with the indicated concentration of PBOX. The percentage of cells in sub- $\mathrm{G}_{1}$ population, $\mathrm{G}_{2} \mathrm{M}$ and those containing a polyploid DNA content are indicated. Data are expressed as mean \pm SE for three independent experiments.

Statistical analysis. The statistical analysis of experimental data was performed using a Student's paired t-test (comparison of two groups) or one-way ANOVA (comparison of more than two groups) were appropriate and results were presented as mean \pm SEM. A value of $\mathrm{P}<0.05$ was considered to be significant.

\section{Results}

PBOX-induced cytotoxicity. The chemical structures of selected PBOXs are illustrated in Fig. 1A. As shown in Fig. 1B, the PBOXs induced a dose-dependent inhibition of cell growth in both the CT-26 and Caco-2 colon adenocarcinoma derived cells with the half-maximal inhibitory concentration $\left(\mathrm{IC}_{50}\right)$ ranging from 0.42 to $11.16 \mu \mathrm{M}$. As anticipated for microtubule targeting drugs the PBOXs induced a dose-dependent increase in cell death (sub- $\mathrm{G}_{1}$ ), $\mathrm{G}_{2} \mathrm{M}$ cell cycle arrest and polyploidy (DNA content $>4 \mathrm{~N}$ ) in both cell lines (Fig. 1C). However, the sub- $\mathrm{G}_{1}$ population induced by $\mathrm{PBOX}$ exposure did not exceed $20 \%$. These data imply an underlying inherent resistance of these cells to the PBOXs.

PBOX-induced protective autophagy in adenocarcinoma cells. Given that autophagy has been associated with resistance to various chemotherapeutics (31) the presence of autophagy in the adherent surviving population of cells following PBOX exposure was next examined. As determined by flow cytometry of acridine orange stained cells, the PBOXs increased the strength of the red fluorescence which is directly proportional to the 
$\mathbf{A}$

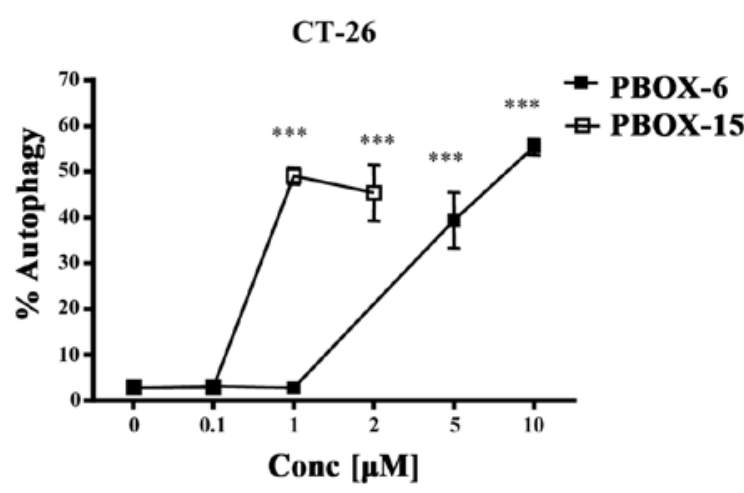

B

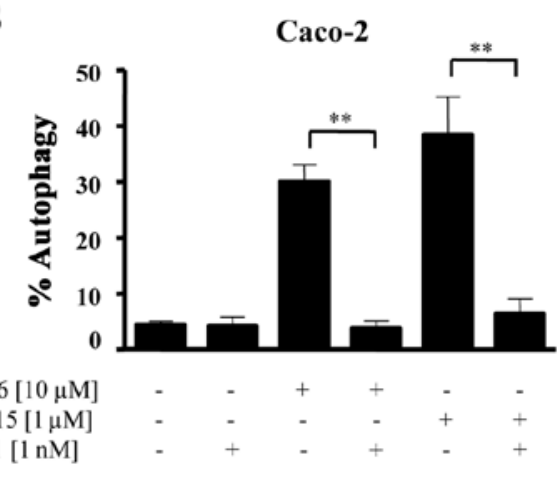

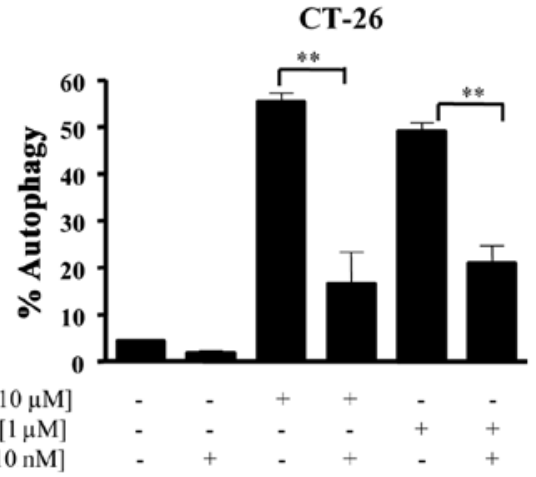

D

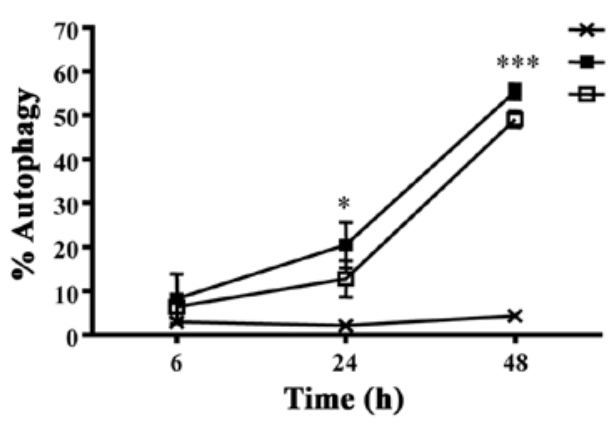

* Control

- PBOX-6
PBOX-6 $[10 \mu \mathrm{M}]$ PBOX-15 [1 $\mu \mathrm{M}]$ BAF-A1 [1 nM]

\section{$\mathbf{C}$ \\ $\mathbf{C}$}

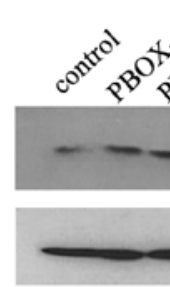

1
Caco-2

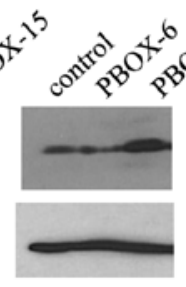

24

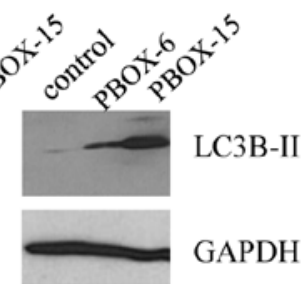

48 (h)
E CT-26

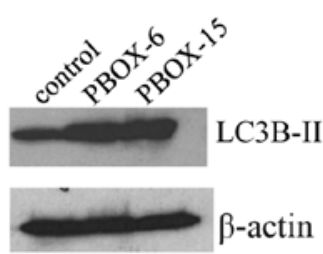

Figure 2. (A, C and E) CT-26 and (B and D) Caco-2 cells were treated with the indicated concentration of PBOX, PBOX-6 (10 $\mu \mathrm{M})$ or PBOX-15 (1 $\mu \mathrm{M})$ for the time indicated in A and D or for (B and C) 48 or (E) 72 h. (A-D) Quantification of PBOX-induced AVO formation was assessed by flow cytometric analysis of acridine orange stained cells using the FL3 mode to quantify the bright red fluorescence/AVO formation and the FL1 mode to quantify the green fluorescence/ uncharged acridine orange. Post-treatment cells were stained with AO. (B and C) BAF-A1 was added 45 min prior to AO staining. Results are expressed as mean values \pm SE of at least 3 independent experiments and analysed, where appropriate, with either the one-way ANOVA test followed by the Benoffi post test or the Student's t-test. ${ }^{* *} \mathrm{P}=0.01 ;{ }^{* * *} \mathrm{P}=0.001$. (D and $\mathrm{E}$ ) Whole cell lysates were probed for LC3B-II, GAPDH or actin by western blot analysis. Results are representative of three independent experiments.

acidity of the cells in a dose dependent manner (Fig. 2A). The intensity of the fluorescence increased over time indicating the development of AVOs after a prolonged exposure to the PBOXs (Fig. 2A). AVOs were also detected by microscopic analysis of acridine orange stained CT-26 cells following a $48 \mathrm{~h}$ exposure to the PBOXs (data not shown). AVOs continued to be detected in the adherent population of cells even after 7 days exposure to the PBOXs further implying a cytoprotective role of autophagy in these cells following prolonged exposure to the PBOXs (data not shown). Numerous studies have demonstrated a dependence of the acidification of cellular organelles on the vacuolar $\mathrm{H}^{+}$
ATPase using the specific inhibitor bafilomycin-A1 (BAF-A1). In agreement with these findings the addition of BAF-A1 prior to acridine orange staining significantly inhibited the formation of AVOs confirming that BAF-A1 can inhibit PBOX-induced late stage autophagy in colon cancer cells (Fig. 2B and C).

LC3, microtubule-associated protein 1 light chain 3, exists as three isoforms (A-C). LC3B-I has been shown to covalently conjugate to phosphatidylethanolamine to form LC3B-II during the formation of autophagosomes. To date, LC3B-II is the only reliable marker to monitor autophagy. LC3B-II is specifically localized to autophagic structures throughout the autophagic 

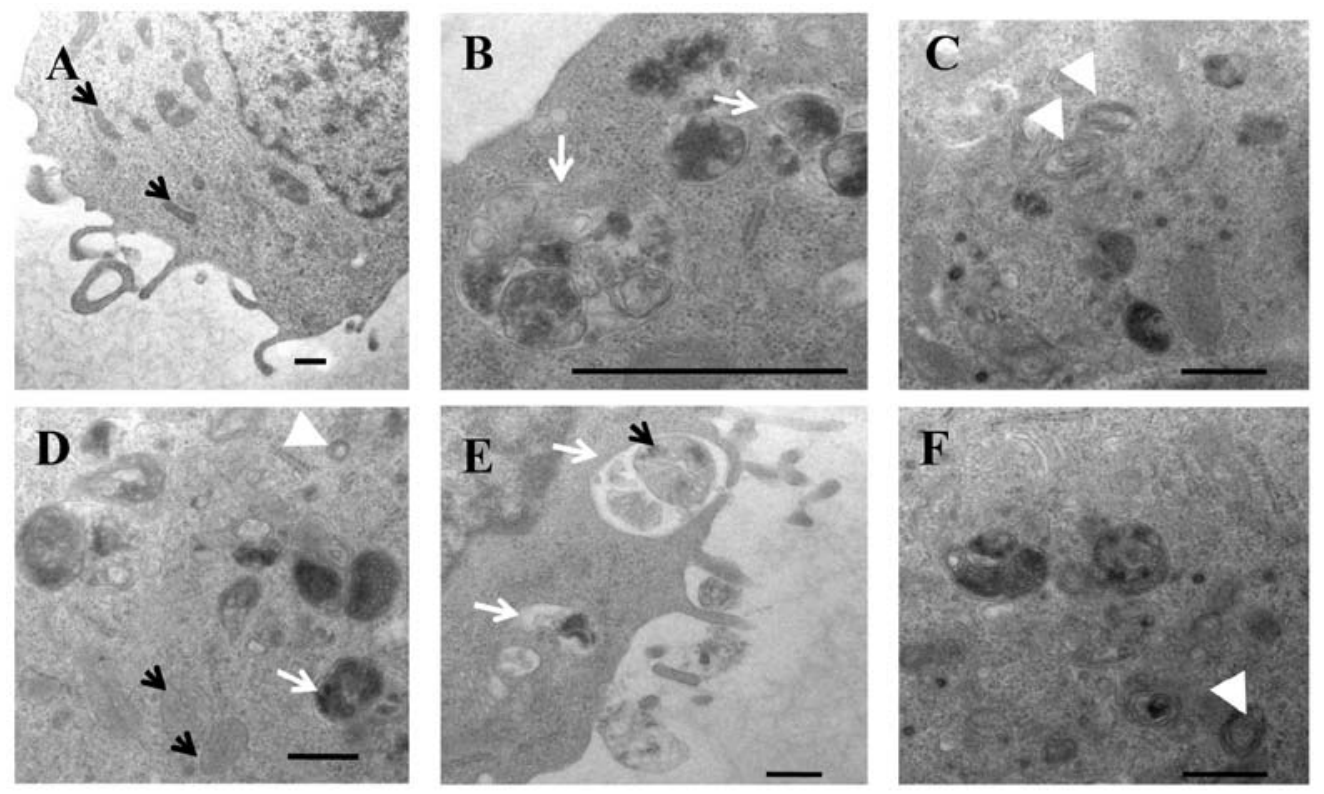

Figure 3. TEM depicted ultrastructures of CT-26 adenocarcinoma cells exposed to PBOX-15. Representative images are shown for cells treated (A) without or (B-F) with PBOX-15 $(1 \mu \mathrm{M})$ for $48 \mathrm{~h}$. Autophagosomes and multivesicular bodies are highlighted by white arrow head. White arrow, autophagolysosomes. Black arrows, point to mitochondria. Bar, $500 \mathrm{~nm}$.

process from phagophore to lysosomal degradation (32). The time-dependent increase in the levels of LC3B-II suggests an increase in the number of autophagosomes in response to PBOXs (Fig. 2D). PBOX-15 was identified as both the most potent antiproliferative PBOX and most effective autophagic initiator in colon cancer cells and was therefore selected for electron microscopy, the gold standard method for determination of autophagy. Prolonged exposure of PBOX-15 increased the formation of autophagosomes and autolysosomes in the surviving adherent population of colon cancer cells (Fig. 3). PBOX-15 induced autolysosomes contained a diverse range of sequestered material which varied from lamellar (including mitochondria derived) to dense granular structures.

The effect of autophagy inhibition on PBOX-induced cell death. We have conclusively demonstrated autophagy in the surviving population of colon cancer cells exposed to PBOXs (Figs. 2 and 3). Ultrastructural analysis clearly demonstrated an accumulation of structures associated with autophagy, such as autophagosomes/multivesicular bodies and autophagolysosomes, in PBOX treated cells. We also demonstrated (Fig. 2B) that BAF-A1 effectively inhibited the late state of autophagy induced by the PBOXs. We next sought to determine whether inhibition of autophagy by BAF-A1 can augment PBOX induced cell death. As shown in Fig. 4A inhibition of late stage autophagy by BAF-A1 enhanced PBOX-6 but not PBOX-15-induced cell death. The increase in cell death due to the combined stimuli of PBOX-6 and BAF-A1 was significantly inhibited by the pan-specific caspase inhibitor Z-VAD suggesting a caspasedependent pathway (Fig. 4B).

BAF-A1 synergistically enhances PBOX-6-induced apoptotic cell death, which associates with a downregulation of the anti-apoptotic protein $\mathrm{Mcl}$-1. Interactions between PBOX-6 and BAF-A1 were further investigated in Caco-2 cells using median dose effect analysis. As shown in Fig. 5A, BAF-A1 significantly enhanced the antiproliferative effect of PBOX-6 in human colon cancer cells as determined using the computer software Calcusyn. A CI value of $<1$ is indicative of synergy. The effects of PBOX-6 and BAF-A 1 combinations were then analysed in relation to expression of both regulators of autophagy and apoptosis. As expected, BAF-A1 alone increased the levels of LC3B-II. It has been postulated that BAF-A1 can increase the levels of LC3B-II by early prevention of its degradation in existing autolysosomes and preventing the fusion of the lysosome with the autophagosome at later stages and is frequently used to assess the authenticity of an autophagic flux (33-35). Levels of PBOX-6 induced LC3B-II where higher in the presence of $1 \mathrm{nM}$ BAF-A1 as compared with BAF-A1 (1 nM) alone indicating an efficient autophagic flux. Levels of PBOX-6 induced LC3B-II did not increase further in the presence of $10 \mathrm{nM}$ BAF-A1 suggesting that the levels of LC3B-II had become saturated at this point. Neither drug alone or in combination influenced the levels of autophagy regulator beclin-1. The effect of the combinations on the lysosomal system was also assessed by western blot analysis of the lysosomal protein LAMP-1. BAF-A1 but not PBOX-6 reduced the levels of unglycosylated LAMP-1 which was reflected in a marginal increase in the levels of glycosylated LAMP-1. BAF-A1-induced change in the glycosylation status of LAMP-1 was maintained in the presence of PBOX-6. We next analysed the effects of the drugs alone and in combination on the Bcl-2 family of anti-apoptotic proteins. High levels of BAF-A1 $(10 \mathrm{nM})$ that induced $\sim 20 \%$ cell death induced the phosphorylation of Bcl-2 and Bcl-xL and reduced the levels of Mcl-1. PBOX-6 alone reduced the levels of Bcl-2 and Mcl-1 and phosphorylated Bcl-xL. BAF-A1 and PBOX-6 combinations reduced the levels of Mcl-1 as compared to either drug alone. 
$\mathbf{A}$

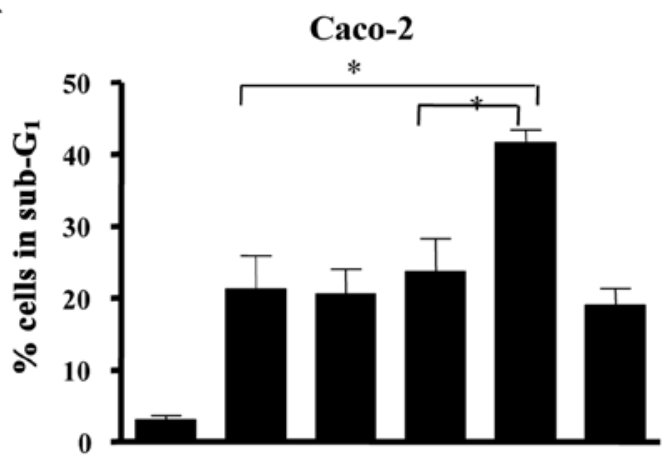

$\begin{array}{lcccccc}\text { PBOX-6 }[10 \mu \mathrm{M}] & - & + & - & - & + & - \\ \text { PBOX-15[1 }[1 \mathrm{M}] & - & - & + & - & - & + \\ \text { BAF-A1 }[10 \mathrm{nM}] & - & - & - & + & + & +\end{array}$

\section{B}

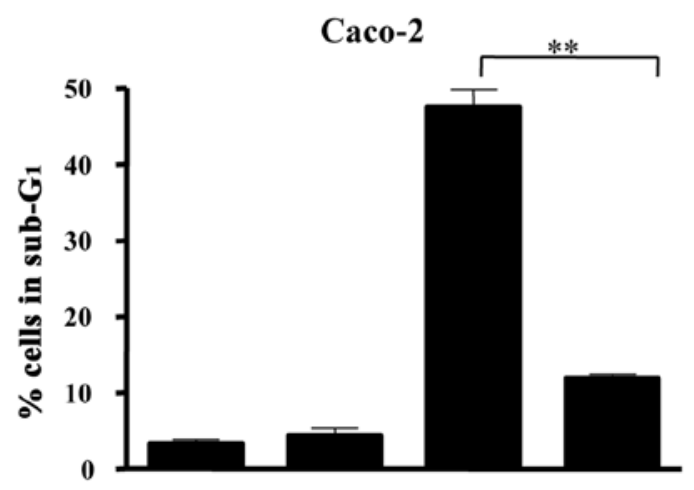

PBOX-6 $[10 \mu \mathrm{M}] \quad-$ BAF-A1 [10 nM] ZVAD $[50 \mu \mathrm{M}]$

Figure 4. (A and B) Caco-2 cells were treated with the indicated drugs alone or in combination for $72 \mathrm{~h}$. The pan-caspase inhibitor Z-VAD was added for $1 \mathrm{~h}$ prior to the addition of the indicated combination of drugs. The percentage of cells in sub- $\mathrm{G}_{1}$ population was analysed by flow cytometric analysis of propidium iodide stained cells. Data are expressed as mean $\pm \mathrm{SE}$ for three independent experiments. ${ }^{*} \mathrm{P}<0.05 ;{ }^{* *} \mathrm{P}<0.01$; Student's t-test.

However, the combined exposure to BAF-A1 and PBOX-6 did not yield any further decrease in the levels of Bcl-2 or Bcl-xL as compared to either drug alone. Finally, BAF-A1 and PBOX-6 combinations increased the extent of PARP cleavage confirming enhanced levels of apoptosis as compared to single drug exposure. Collectively, our results demonstrate that the inhibition of autophagy synergistically sensitises colon cancer cells to PBOX-6.

\section{Discussion}

The PBOXs are a novel class of microtubule targeting agents (MTAs) which are structurally distinct from MTAs currently used within the clinic. The PBOXs have demonstrated promising anticancer activity in ex vivo patient samples and in vivo tumour models $(5,8,9,36)$. However, as with all MTAs a significant proportion of cells remain viable following prolonged exposure to the PBOXs. For this reason alternative approaches that combine the PBOXs with a second agent exhibiting a different mechanism of action is required to further improve the therapeutic potential of the PBOXs. We have previously published preclinical data demonstrating successful combinations of the PBOXs with the tyrosine kinase inhibitor, imatinib mesylate $(37,38)$, the cyclin dependent kinase inhibitor, flavopiridol $(39,40)$ and also with the tumour necrosis factor-related apoptosis-inducing ligand (36). Furthermore, we have demonstrated potential clinical benefits in combining the PBOXs with radiotherapy for the treatment of prostate cancer (41).

In this study, we report novel findings demonstrating that the tubulin-targeting PBOX-6 induces both apoptosis and autophagy in Caco-2 cells, highlighting autophagy as a novel therapeutic target which could potentially augment PBOX induced cell death. Activation of autophagy has long been recognised as a cellular response to stress, in particular nutrient deprivation. Recent data suggest autophagy is induced as a pro-survival strategy to many anticancer therapies. The cytoprotective role of autophagy to chemotherapeutics has generated immense interest in the potential to target autophagy as an adjuvant therapy.

The result of autophagy manipulation is dependent on the combined influence of several factors including cell type, autophagy initiator, the combined stimuli to the autophagy initiator and selected combination. Given the varied and complex outcome of autophagy manipulation it is not yet fully understood how to effectively modulate autophagy to enhance the therapeutic efficacy of individual anticancer therapies. Furthermore, as autophagy functions as regulator of homeostasis the use of autophagy inhibitors as an adjunctive therapy in organs in which autophagy plays a homeostatic role may yield undesirable side-effects (42). Hence given the infancy of autophagy manipulation as a novel anticancer strategy there is an urgent need to accumulate preclinical data on a broad spectrum of chemotherapeutics in various cell types and in in vivo models.

Herein we present novel significant findings demonstrating that inhibition of late stage autophagy by BAF-A1 enhanced PBOX-6 but not PBOX-15-induced apoptotic death. Recent studies demonstrated unique autophagy properties endowed by a C, D-spirolactone analogue of paclitaxel not shared with the parent compound highlighting the complexity of autophagy stimulation (43). Inhibition of the early stages of autophagy by 3-methyladenine did not augment PBOX-induced cell death (data not shown). Similarly, other studies have demonstrated therapeutic benefits in inhibiting late stage but not early stage autophagy in combination with anticancer agents (44). These results accentuate the varied and complex outcome of autophagy manipulation warranting continued research into autophagy manipulation as an adjuvant therapy.

In concurrence with independent research carried out in different colon cancer derived cells (24) we found that inhibition of autophagy with BAF-A1 induced apoptotic cell death which was associated with cleavage of caspase-3 and PARP. In this report we present novel findings demonstrating that BAF-A1-induced apoptosis was associated with changes to the Bcl-2 family of anti-apoptotic proteins; namely phosphoryla- 


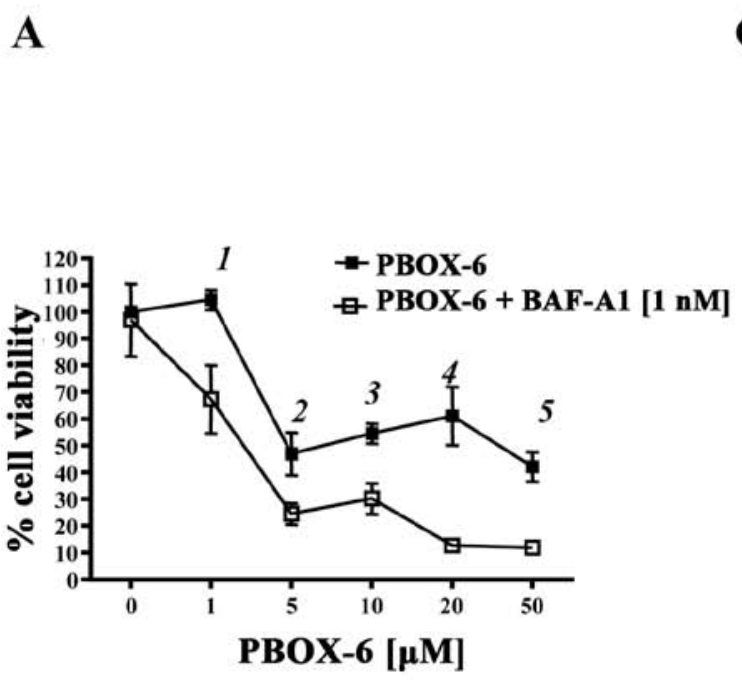

B

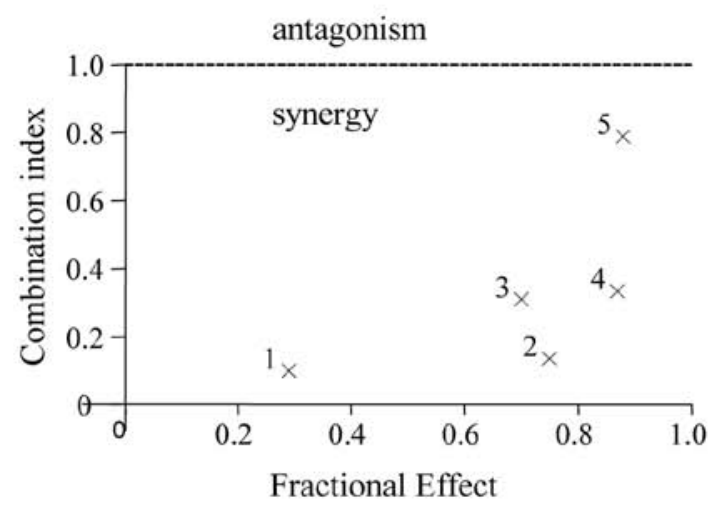

C
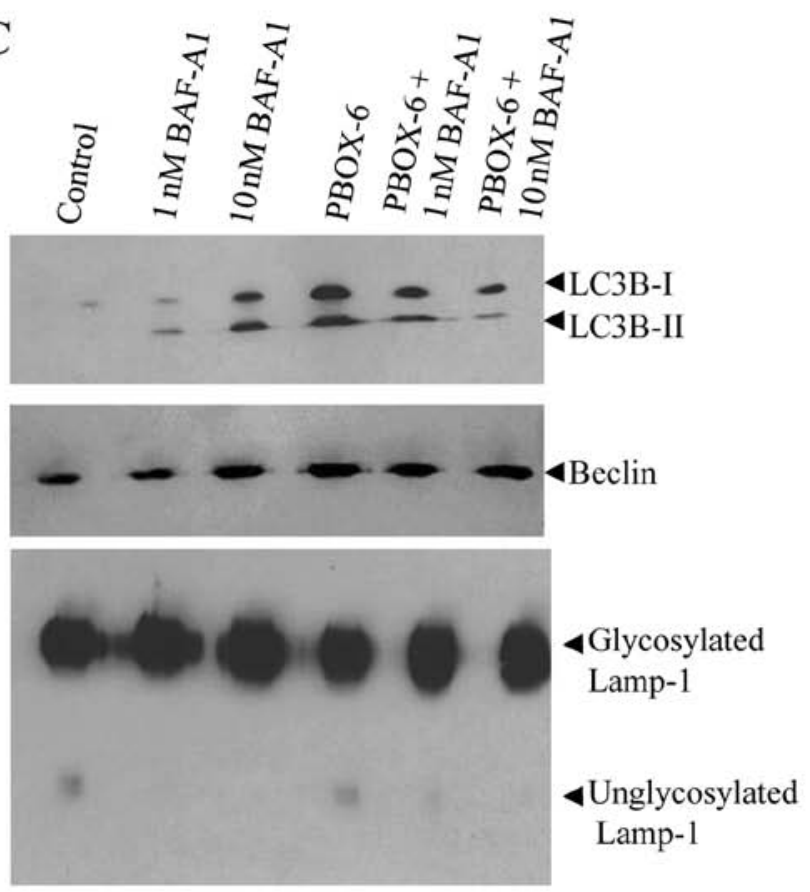

$4 \mathrm{Mcl}-1$
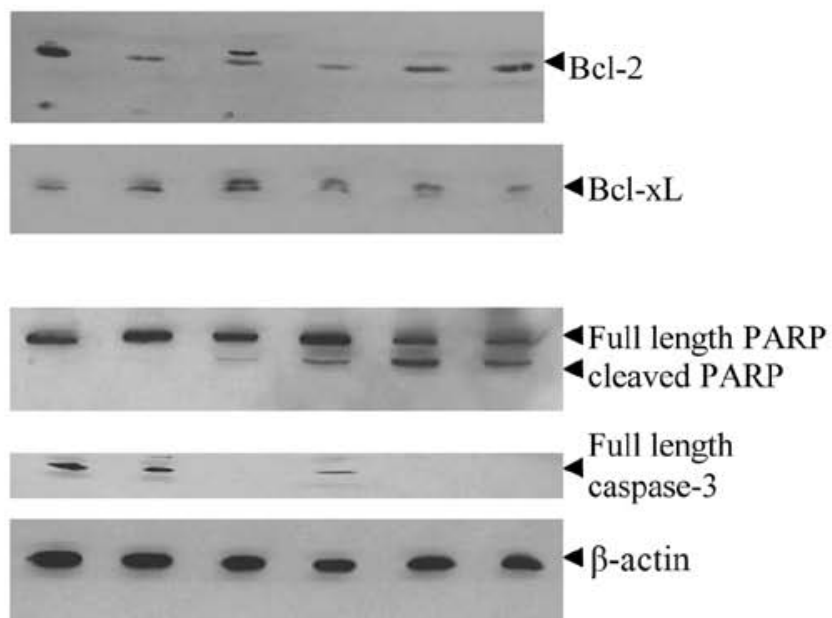

Figure 5. The autophagy inhibitor BAF-A1 synergistically enhances the apoptotic effect of PBOX-6 in human adenocarcinoma derived cells. (A) Caco-2 cells were exposed to the indicated concentration of PBOX-6 in the presence or absence of BAF-A1 ( $1 \mathrm{nM})$ for $72 \mathrm{~h}$. Cell viability was assessed by the Alamar blue assay. Data are expressed as mean \pm SE. (B) Combination index values for each fraction affected were determined using the commercially available Calcusyn software (Biosoft, UK). A combination index value $>1,=1,<1$ indicates antagonism, an additive effect and synergy, respectively. Combinations analysed are marked in bold italics in A. (C) Western blot analysis of whole cell lysates from selected combinations indicated in A.

tion of Bcl-2 and Bcl-xL and downregulation of Mcl-1. These findings compliment other studies demonstrating that BAF-A1 can overcome Bcl-xL mediated chemoresistance in small cell lung carcinoma cells by restoration of a caspase-independent apoptotic pathway (45). Similarly, in the mouse insulinoma M1N6 cell line, BAF-A1 reduced Bcl-2 expression coincided with a reduction in cell viability (46). Other autophagy inhibitors 3-MA and chloroquine induced caspase-dependent apoptosis which was associated with the downregulation of Mcl-1 (47). It was also postulated that interactions between Bcl-2 family members and ATG12 may constitute an important point between autophagy and apoptosis in response to specific signals (48). Also, Bcl-2L11/BIM was recently identified as a novel molecular link between autophagy and apoptosis (49). Taken together these findings suggest that apoptosis induced following the inhibition of autophagy associates with changes in the expression of members of the Bcl-2 family of anti-apoptotic proteins. PBOX-6 induced apoptosis was also associated with a downregulation of Mcl-1. Furthermore, co-treatment with BAF-A1 enhanced PBOX-6 induced apoptosis which associated with a further decline in Mcl-1 protein levels. These findings provide a rationale for combining agents which target $\mathrm{Mcl}-1$ in combination with autophagy inhibitors for the treatment of human cancer. 
We also report a novel finding demonstrating that BAF-A1 reduced the levels of unglycosylated LAMP-1. LAMP proteins are required for the mobility of lysosomes and their fusion with phagosomes (50). Glycosylation of LAMP-1 is important for the stability of LAMP-1 in the lysosomal membrane (51). Inhibition of Abl-kinase signaling by imatinib was shown to alter LAMP-1 glycosylation in A549 lung carcinoma cell line (52). Furthermore, following imatinib exposure the increase in the glycosylated form of LAMP-1 associated with decreased autophagy (52). Here we show that inhibition of autophagy by BAF-A1 decreases the amount of the unstable unglycosylated LAMP-1 which coincided with a marginal increase in the glycosylated stable form of LAMP-1. Together these findings suggest that inhibition of autophagy associates with changes in the glycosylation status of LAMP-1.

In summary, we have demonstrated novel findings showing that the PBOXs induce apoptosis and autophagy in colon cancer cells. Blocking autophagy in the late stages represents a novel strategy to augment PBOX-6-induced lethality in these cells. Autophagy plays a cytoprotective role in response to PBOX therapy therefore further studies are warranted to delineate what components of the autophagic pathway exhibit a cytoprotective function to further enhance the inherent chemotherapeutic properties of the PBOXs.

\section{Acknowledgements}

We would like to thank the Health Research Board Ireland for funding the project.

\section{References}

1. Campiani G, Nacci V, Fiorini I, De Filippis MP, Garofalo A, Ciani SM, Greco G, Novellino E, Williams DC, Zisterer DM, et al: Synthesis, biological activity, and SARs of pyrrolobenzoxazepine derivatives, a new class of specific 'peripheral-type' benzodiazepine receptor ligands. J Med Chem 39: 3435-3450, 1996.

2. Zisterer DM, McGee MM, Campiani G, Ramunno A, Fattorusso C, Nacci V, Lawler M and Williams DC: Pyrrolo1,5-benzoxazepines: a new class of apoptotic agents. Biochem Soc Trans 29: 704-706, 2001.

3. Mulligan JM, Greene LM, Cloonan S, McGee MM, Onnis V, Campiani G, Fattorusso C, Lawler M, Williams DC and Zisterer DM: Identification of tubulin as the molecular target of proapoptotic pyrrolo-1,5-benzoxazepines. Mol Pharmacol 70: 60-70, 2006.

4. McGee MM, Greene LM, Ledwidge S, Campiani G, Nacci V, Lawler M, Williams DC and Zisterer DM: Selective induction of apoptosis by the pyrrolo-1,5-benzoxazepine 7-[[dimethylcarbamoyl]oxy]-6-(2-naphthyl)pyrrolo-[2,1-d] (1,5)-benzoxazepine (PBOX-6) in leukemia cells occurs via the c-Jun NH2-terminal kinase-dependent phosphorylation and inactivation of Bcl-2 and Bcl-XL. J Pharmacol Exp Ther 310: 1084-1095, 2004.

5. Greene LM, Fleeton M, Mulligan J, Gowda C, Sheahan BJ, Atkins GJ, Campiani G, Nacci V, Lawler M, Williams DC, et al: The pyrrolo-1, 5-benzoxazepine, PBOX-6, inhibits the growth of breast cancer cells in vitro independent of estrogen receptor status and inhibits breast tumour growth in vivo. Oncol Rep 14: 1357-1363, 2005.

6. Greene LM, Campiani G, Lawler M, Williams DC and Zisterer DM: BubR1 is required for a sustained mitotic spindle checkpoint arrest in human cancer cells treated with tubulin-targeting pyrrolo-1,5-benzoxazepines. Mol Pharmacol 73: 419-430, 2008 .

7. Nathwani SM, Butler S, Fayne D, McGovern NN, Sarkadi B, Meegan MJ, Lloyd DG, Campiani G, Lawler M, Williams DC, et al: Novel microtubule-targeting agents, pyrrolo-1,5-benzoxazepines, induce apoptosis in multi-drug-resistant cancer cells. Cancer Chemother Pharmacol 66: 585-596, 2010.
8. McElligott AM, Maginn EN, Greene LM, McGuckin S, Hayat A, Browne PV, Butini S, Campiani G, Catherwood MA, Vandenberghe E, et al: The novel tubulin-targeting agent pyrrolo-1,5-benzoxazepine-15 induces apoptosis in poor prognostic subgroups of chronic lymphocytic leukemia. Cancer Res 69: 8366-8375, 2009.

9. Bright SA, McElligott AM, O'Connell JW, O'Connor L, Carroll P, Campiani G, Deininger MW, Conneally E, Lawler M, Williams DC, et al: Novel pyrolo-1,5-benzoxazepine compounds display significant activity against resistant chronic myeloid leukaemia cells in vitro, in ex vivo patient samples and in vivo. Br J Cancer 102: 1474-1482, 2010.

10. Nathwani SM, Butler S, Meegan MJ, Campiani G, Lawler M, Williams DC and Zisterer DM: Dual targeting of tumour cells and host endothelial cells by novel microtubule-targeting agents, pyrrolo-1,5-benzoxazepines. Cancer Chemother Pharmacol 65: 289-300, 2010.

11. Sato K, Tsuchihara K, Fujii S, Sugiyama M, Goya T, Atomi Y, Ueno T, Ochiai A and Esumi H: Autophagy is activated in colorectal cancer cells and contributes to the tolerance to nutrient deprivation. Cancer Res 67: 9677-9684, 2007.

12. Wu YC, Wang XJ, Yu L, Chan FK, Cheng AS, Yu J, Sung JJ, Wu WK and Cho CH: Hydrogen sulfide lowers proliferation and induces protective autophagy in colon epithelial cells. PLoS One 7: e37572, 2012.

13. Li J, Hou N, Faried A, Tsutsumi S and Kuwano H: Inhibition of autophagy augments 5-fluorouracil chemotherapy in human colon cancer in vitro and in vivo model. Eur J Cancer 46: 1900-1909, 2010

14. Li DD, Sun T, Wu XQ, Chen SP, Deng R, Jiang S, Feng GK, Pan JX, Zhang XS, Zeng YX, et al: The inhibition of autophagy sensitises colon cancer cells with wild-type p53 but not mutant p53 to topotecan treatment. PLoS One 7: e45058, 2012.

15. Paillas S, Causse A, Marzi L, de Medina P, Poirot M, Denis V, Vezzio-Vie N, Espert L, Arzouk H, Coquelle A, et al: MAPK14/p38alpha confers irinotecan resistance to TP53-defective cells by inducing survival autophagy. Autophagy 8: 1098-1112, 2012.

16. Ravikumar B, Sarkar S, Davies JE, Futter M, GarciaArencibia M, Green-Thompson ZW, Jimenez-Sanchez M, Korolchuk VI, Lichtenberg M, Luo S, et al: Regulation of mammalian autophagy in physiology and pathophysiology. Physiol Rev 90: 1383-1435, 2010.

17. Han W, Sun J, Feng L, Wang K, Li D, Pan Q, Chen Y, Jin W, Wang X, Pan H, et al: Autophagy inhibition enhances daunorubicin-induced apoptosis in K562 cells. PLoS One 6: e28491, 2011.

18. Shen S, Kepp O and Kroemer G: The end of autophagic cell death? Autophagy 8: 1-3, 2012.

19. Greene LM, O'Boyle NM, Nolan DP, Meegan MJ and Zisterer DM: The vascular targeting agent Combretastatin-A4 directly induces autophagy in adenocarcinoma-derived colon cancer cells. Biochem Pharmacol 84: 612-624, 2012.

20. Cook KL, Shajahan AN and Clarke R: Autophagy and endocrine resistance in breast cancer. Expert Rev Anticancer Ther 11: 1283-1294, 2011.

21. Zois CE and Koukourakis MI: Radiation-induced autophagy in normal and cancer cells: towards novel cytoprotection and radio-sensitization policies? Autophagy 5: 442-450, 2009.

22. Clarke PG and Puyal J: Autophagic cell death exists. Autophagy 8: 867-869, 2012.

23. Boya P, Gonzalez-Polo RA, Casares N, Perfettini JL, Dessen P, Larochette N, Metivier D, Meley D, Souquere S, Yoshimori T, et al: Inhibition of macroautophagy triggers apoptosis. Mol Cell Biol 25: 1025-1040, 2005.

24. Wu YC, Wu WK, Li Y, Yu L, Li ZJ, Wong CC, Li HT, Sung JJ and Cho $\mathrm{CH}$ : Inhibition of macroautophagy by bafilomycin A1 lowers proliferation and induces apoptosis in colon cancer cells. Biochem Biophys Res Commun 382: 451-456, 2009.

25. Bing L, Dejuan K, Yang L, Nan L, Mengzi H, Shumei M and Xiaodong L: Autophagy inhibition plays the synergetic killing roles with radiation in the multi-drug resistant SK VCR ovarian cancer cells. Radiat Oncol 7: 213, 2012.

26. Zhu S, Cao L, Yu Y, Yang L, Yang M, Liu K, Huang J, Kang R, Livesey KM and Tang D: Inhibiting autophagy potentiates the anticancer activity of IFN $\alpha$ in chronic myeloid leukemia cells. Autophagy 9: 317-327, 2012.

27. Swampillai AL, Salomoni $P$ and Short SC: The role of autophagy in clinical practice. Clin Oncol (R Coll Radiol) 24: 387-395, 2012. 
28. Garcia-Echeverria C and Sellers WR: Drug discovery approaches targeting the PI3K/Akt pathway in cancer. Oncogene 27: 5511-5526, 2008.

29. Cerniglia GJ, Karar J, Tyagi S, Christofidou-Solomidou M, Rengan R, Koumenis C and Maity A: Inhibition of autophagy as a strategy to augment radiosensitization by the dual phosphatidylinositol 3-kinase/mammalian target of rapamycin inhibitor NVP-BEZ235. Mol Pharmacol 82: 1230-1240, 2012.

30. Yang ZJ, Chee CE, Huang S and Sinicrope FA: The role of autophagy in cancer: therapeutic implications. Mol Cancer Ther 10: 1533-1541, 2011.

31. Maycotte P and Thorburn A: Autophagy and cancer therapy. Cancer Biol Ther 11: 127-137, 2011.

32. Nakatogawa H, Suzuki K, Kamada Y and Ohsumi Y: Dynamics and diversity in autophagy mechanisms: lessons from yeast. Nat Rev Mol Cell Biol 10: 458-467, 2009.

33. Yamamoto A, Tagawa Y, Yoshimori T, Moriyama Y, Masaki R and Tashiro Y: Bafilomycin A1 prevents maturation of autophagic vacuoles by inhibiting fusion between autophagosomes and lysosomes in rat hepatoma cell line, H-4-II-E cells. Cell Struct Funct 23: 33-42, 1998.

34. Jahreiss L, Menzies FM and Rubinsztein DC: The itinerary of autophagosomes: from peripheral formation to kiss-and-run fusion with lysosomes. Traffic 9: 574-587, 2008

35. Klionsky DJ, Elazar Z, Seglen PO and Rubinsztein DC: Does bafilomycin A1 block the fusion of autophagosomes with lysosomes? Autophagy 4: 849-950, 2008.

36. Maginn EN, Browne PV, Hayden P, Vandenberghe E, MacDonagh B, Evans P, Goodyer M, Tewari P, Campiani G, Butini S, et al: $\mathrm{PBOX}-15$, a novel microtubule targeting agent, induces apoptosis, upregulates death receptors, and potentiates TRAIL-mediated apoptosis in multiple myeloma cells. Br J Cancer 104: 281-289, 2011.

37. Greene LM, Kelly L, Onnis V, Campiani G, Lawler M, Williams DC and Zisterer DM: STI-571 (imatinib mesylate) enhances the apoptotic efficacy of pyrrolo-1,5-benzoxazepine-6, a novel microtubule-targeting agent, in both STI-571-sensitive and -resistant Bcr-Abl-positive human chronic myeloid leukemia cells. J Pharmacol Exp Ther 321: 288-297, 2007.

38. Bright SA, Greene LM, Greene TF, Campiani G, Butini S, Brindisi M, Lawler M, Meegan MJ, Williams DC and Zisterer DM: The novel pyrrolo-1, 5-benzoxazepine, PBOX-21, potentiates the apoptotic efficacy of STI571 (imatinib mesylate) in human chronic myeloid leukaemia cells. Biochem Pharmacol 77: 310-321, 2009

39. Bright SA, Campiani G, Deininger MW, Lawler M, Williams DC and Zisterer DM: Sequential treatment with flavopiridol synergistically enhances pyrrolo-1,5-benzoxazepine-induced apoptosis in human chronic myeloid leukaemia cells including those resistant to imatinib treatment. Biochem Pharmacol 80 $31-38,2010$.
40. Nathwani SM, Cloonan SM, Stronach M, Campiani G, Lawler M, Williams DC and Zisterer DM: Novel microtubuletargeting agents, pyrrolo-1,5-benzoxazepines, induce cell cycle arrest and apoptosis in prostate cancer cells. Oncol Rep 24: 1499-1507, 2010.

41. Forde JC, Maginn EN, McNamara G, Martin LM, Campiani G, Williams DC, Zisterer D, McElligott AM, Lawler M, Lynch TH, et al: Microtubule-targeting-compound PBOX-15 radiosensitizes cancer cells in vitro. Cancer Biol Ther 11: 421-428, 2011.

42. Kimura T, Takabatake Y, Takahashi A and Isaka Y: Chloroquine in cancer therapy: a double-edged sword of autophagy. Cancer Res 73: 3-7, 2013.

43. Trmcic MV, Matovic RV, Tovilovic GI, Ristic BZ, Trajkovic VS, Ferjancic ZB and Saicic RN: A novel C,D-spirolactone analogue of paclitaxel: autophagy instead of apoptosis as a previously unknown mechanism of cytotoxic action for taxoids. Org Biomol Chem 25: 4933-4942, 2012.

44. Kanematsu S, Uehara N, Miki H, Yoshizawa K, Kawanaka A Yuri $\mathrm{T}$ and Tsubura A: Autophagy inhibition enhances sulforaphane-induced apoptosis in human breast cancer cells. Anticancer Res 30: 3381-3390, 2010.

45. Sasazawa Y, Futamura Y, Tashiro E and Imoto M: Vacuolar $\mathrm{H}^{+}$-ATPase inhibitors overcome Bcl-xL-mediated chemoresistance through restoration of a caspase-independent apoptotic pathway. Cancer Sci 100: 1460-1467, 2009.

46. Hettiarachchi KD, Zimmet PZ and Myers MA: The plecomacrolide vacuolar-ATPase inhibitor bafilomycin, alters insulin signaling in MIN6 beta-cells. Cell Biol Toxicol 22: 169-181, 2006.

47. Pliyev BK and Menshikov M: Differential effects of the autophagy inhibitors 3-methyladenine and chloroquine on spontaneous and TNF-alpha-induced neutrophil apoptosis. Apoptosis 17: 1050-1065, 2012.

48. Rubinstein AD, Eisenstein M, Ber Y, Bialik S and Kimchi A: The autophagy protein Atg12 associates with antiapoptotic Bcl-2 family members to promote mitochondrial apoptosis. Mol Cell 44: 698-709, 2011.

49. Luo S and Rubinsztein DC: BCL2L11/BIM: A novel molecular link between autophagy and apoptosis. Autophagy 9: 104-105, 2013.

50. Huynh KK, Eskelinen EL, Scott CC, Malevanets A, Saftig P and Grinstein S: LAMP proteins are required for fusion of lysosomes with phagosomes. EMBO J 26: 313-324, 2007.

51. Kundra R and Kornfeld S: Asparagine-linked oligosaccharides protect Lamp-1 and Lamp-2 from intracellular proteolysis. J Biol Chem 274: 31039-31046, 1999.

52. Yogalingam G and Pendergast AM: Abl kinases regulate autophagy by promoting the trafficking and function of lysosomal components. J Biol Chem 283: 35941-35953, 2008. 